\title{
Dietary energy density: estimates, trends and dietary determinants for a nationally representative sample of the Irish population (aged 5-90 years)
}

\author{
Laura O'Connor ${ }^{1,2 *}$, Janette Walton ${ }^{1}$ and Albert Flynn ${ }^{1}$ \\ ${ }^{1}$ School of Food and Nutritional Science, University College Cork, Cork, Republic of Ireland \\ ${ }^{2}$ MRC Epidemiology Unit, School of Clinical Medicine, Institute of Metabolic Sciences, University of Cambridge, Box 285, \\ Cambridge Biomedical Campus, Cambridge CB2 OQQ, UK
}

(Submitted 10 June 2014 - Final revision received 3 September 2014 - Accepted 1 October 2014 - First published online 17 November 2014)

\begin{abstract}
Higher dietary energy density (DED) has been reported to be associated with weight gain, obesity and poorer dietary quality, yet nationally representative estimates that would allow tracking of secular trends and inter-country comparisons are limited. The aims of the present study were to calculate DED estimates for the Irish population and to identify dietary determinants of DED. Weighed/semi-weighed food records from three cross-sectional surveys (the National Children's Food Survey, the National Teens' Food Survey and the National Adult Nutrition Survey) were collated to estimate habitual dietary intakes for a nationally representative sample of the Irish population, aged 5-90 years ( $n$ 2535). DED estimates, calculated using the total diet method, the food only method and a novel method, including foods and solids in beverages, were 3.70 (SD 1.09), 7.58 (SD 1.72) and 8.40 (SD 1.88) kJ/g, respectively. Determinants of DED did not vary by the calculation method used. Variation in the intakes of fruit, vegetables and sugar-sweetened beverages (SSB) across consumer groups contributed to the largest variance in DED estimates, followed by variation in the intakes of potatoes, fresh meat, bread, chips, ready-to-eat breakfast cereals, and confectionery. DED estimates were inversely associated with age group and consistently lower for females than for males. The inverse association of DED with age group was explained by higher intakes of vegetables, fruit, fish, potatoes, fresh meat and brown bread and lower intakes of SSB, chocolate confectionery, ready-to-eat breakfast cereals and savoury snacks in older age groups. Females consumed, on average, 1.5 times more fruit and vegetables combined when compared with males, largely explaining the sex differences in DED estimates. Current DED estimates for adults were similar to those calculated in a previous survey, carried out 10 years earlier. These estimates and determinants serve as a baseline for comparison for other works and public health campaigns.
\end{abstract}

Key words: Energy density: Dietary determinants: Nationally representative estimates: Trends: Children: Adolescents: Adults

A solid body of evidence exists from experimental investigations and intervention studies for the association of dietary energy density (DED) with weight gain, increased energy intake (EI) and obesity ${ }^{(1-3)}$. Body weight status $^{(4)}, \mathrm{BMI}^{(5)}$, waist circumference ${ }^{(6)}$, obesity and the metabolic syndrome ${ }^{(7)}$ have all been found to be positively associated with DED in cross-sectional studies, providing evidence that the observations extend to free-living populations. A relationship between DED and the nutritional quality of the diet has also been highlighted; lower DED has been reported to be associated with higher dietary quality in Irish children and teenagers, American adults, Spanish adults and elderly Spanish adults ${ }^{(8-11)}$ and with intakes of foods and nutrients related to better dietary quality in Mediterranean, Australian and American adults ${ }^{(12-15)}$. DED has also been proposed as a potential proxy for dietary quality ${ }^{(16)}$, and low DED has been reported to be associated with a trend towards a healthier lifestyle, including lower alcohol consumption, smoking and physical inactivity, in a representative sample of Spanish adults ${ }^{(10)}$.

To date, there have been no estimations of DED in an Irish population. As DED is associated with dietary quality and with obesity, it is worth estimating DED values for the population. This will allow tracking of secular trends and enable comparison with those of other countries. It is also worth distinguishing the dietary components that influence DED in free-living populations to explain differences in DED estimates and to ascertain potential targets for public health messages to lower DED estimates. The National Children's Food Survey (NCFS), the National Teens' Food Survey (NTFS) and the

Abbreviations: \%TE, percentage of total energy; DED, dietary energy density; EI, energy intake; NANS, National Adult Nutrition Survey; NCFS, National Children's Food Survey; NSIFCS, North/South Ireland Food Consumption Survey; NTFS, National Teens' Food Survey; RTEBC, ready-to-eat breakfast cereals; SSB, sugar-sweetened beverages; UR, under-reporters for energy.

* Corresponding author: L. O'Connor, email laura.oconnor@mrc-epid.cam.ac.uk 
National Adult Nutrition Survey (NANS) together form a nationally representative sample of the Irish population aged 5-90 years that provides fully disaggregated habitual food consumption data at the level of the individual from which calculation of such estimates is possible.

The aims of the present study were to calculate and present DED estimates for a nationally representative sample of the Irish population and to identify dietary determinants of DED in a free-living population.

\section{Methods}

\section{Study sample and dietary intake assessment}

The NCFS (2003-4), the NTFS (2005-6) and the NANS (2008-10) were carried out to establish databases of habitual food and drink consumption in representative samples of Irish children, adolescents and adults aged 5-12, 13-17 and 18-90 years, respectively. A $7 \mathrm{~d}$ weighed food record was used to collect dietary intake data from 594 children (293 boys and 301 girls), a $7 \mathrm{~d}$ semi-weighed food record was used to collect dietary intake data from 441 adolescents (224 boys and 217 girls), and a $4 \mathrm{~d}$ semi-weighed food record was used to collect dietary intake data from 1500 adults (760 females and 740 males). Analyses of dietary intake data were carried out using WISP ${ }^{\odot}$ (Tinuviel Software), which contains McCance and Widdowson's The Composition of Foods, 6th edition ${ }^{(17)}$, 5th edition ${ }^{(18)}$ plus supplemental volumes ${ }^{(19-28)}$ and the Irish Food Composition Database ${ }^{(29)}$. Ethical approval was obtained from St James' Hospital and Federated Dublin Voluntary Hospitals Joint Research Ethics Committee for the NCFS and the University College Cork Clinical Research Ethics Committee of the Cork Teaching Hospitals for the NTFS and the NANS. Written consent was obtained from the participants or from the parents/guardians for participants aged $<18$ years. A full description of methodologies is given elsewhere (http://www.iuna.net).

\section{Estimation of dietary energy density}

DED was estimated as $\mathrm{kJ} / \mathrm{g}$ and was calculated using three different methods by taking the following into account: (1) energy and weight of the total diet; (2) energy and weight including only foods and excluding all beverages (i.e. all solid foods and liquid-like foods such as yogurts and soups included and all beverages excluded); (3) energy and weight of all foods and solids in beverages (values for solids in beverages were calculated by removing the water portion of each food code).

\section{Estimation of dietary energy density accounting for under-reporters for energy}

DED was estimated both including and excluding underreporters for energy (UR). For each child and teenager, BMR was predicted from standard equations using body weight and height ${ }^{(30)}$. Minimum EI cut-off points, calculated as multiples of $\mathrm{BMR}^{(31)}$, were used to identify UR. In the study sample, $32 \%$ of children and $64 \%$ of teenagers were classified as UR.

In adults, body composition was measured using the Tanita body composition analyser BC-420MA, which calculated BMR through multiple regression analysis using fat-free mass values (Tanita Limited). EI is expressed as a ratio of BMR (EI:BMR). The cut-off limits proposed by Goldberg et al. ${ }^{(32)}$ to classify individuals as UR were used. The cut-off point relevant to the NANS was $1 \cdot 10 ; 30 \%$ of the participants had an EI:BMR below this value.

\section{Statistical analyses}

Statistical analyses were conducted using SPSS $^{\circledR}$ version 15.0 for Windows ${ }^{\mathrm{TM}}$ (SPSS, Inc.). DED was estimated and expressed as mean, median and standard deviation by sex and age group. An independent-samples $t$ test and a two-way between-groups ANOVA were conducted to evaluate differences in DED estimates by sex and age group.

The dietary determinants of DED were identified by categorising the participants by tertile of energy, macronutrient and food group intakes into low-, medium- and high- or non-, low- and high-consumer groups of each. The participants were categorised into consumers and non-consumers for some less consumed food groups. A one-way ANOVA or an independent-samples $t$ test was used to test for significant differences $(P<0.05)$ in means across consumer groups. Significant differences between the groups were determined using ANOVA followed by Tukey's honestly significant difference or Hochberg's GT2 post hoc test where appropriate.

Post hoc tests were chosen depending on similarity between sample sizes and homogeneity of variance between the groups (as determined by Levene's test). The effect size of the significant differences between consumer groups was calculated as follows: $\eta^{2}=$ sum of squares between groups/total sum of squares (values $\geq 0.01$ indicate a small effect, $\geq 0.06 \mathrm{a}$ medium effect and $\geq 0.14$ a large effect, as classified by Cohen $^{(33)}$ ).

Food intake patterns that may explain the difference in DED estimates between sexes and with age were estimated by examining food group intakes across age groups in males and females separately. For this purpose, the participants were categorised into three age groups: 5-18; 19-50; 51-90 years. ANOVA was used to test for significant differences $(P<0.05)$ in mean intakes across age groups. Significant differences between the groups were determined using ANOVA followed by either Tukey's honestly significant difference or Hochberg's GT2 post hoc test. A one-way between-groups ANCOVA was used to test for significant differences between sexes adjusted for age.

\section{Secular trends and generational effects}

The DED estimates of a subsample from the NANS (those aged 18-64 years, $n$ 1274) were compared with estimates calculated for the participants of the North/South Ireland Food Consumption Survey (NSIFCS), 2001 ( $n$ 1379). The NSIFCS was carried out 10 years before the NANS and included a 
representative sample of adults aged 18-64 years living in Ireland. Dietary intake data were collected using a $7 \mathrm{~d}$ food record. A full description of the survey design and methodology of the NSIFCS has been published previously ${ }^{(34)}$. Differences in DED estimates calculated for the NANS and NSIFCS were examined using a one-way betweengroups ANCOVA. This comparison also allowed to assess the possibility of a generational effect on trends observed between DED and age groups in the cross-sectional analysis.

\section{Results}

For each of the three methods, DED estimates were inversely associated with age group $(P$ linear trend $<0 \cdot 001)$ and females had lower mean DED estimates than males $(P<0 \cdot 001)$ (Table 1$)$. DED estimates were lower for females in all age groups $(P<0 \cdot 001)$.

Mean DED estimates calculated excluding UR (see online supplementary Table S1) were similar to those calculated for the total population and trended similarly by sex and across age groups. Exclusion of UR resulted in estimates similar to those calculated for the total population regardless of the calculation method used.

Determinants of DED did not vary by the calculation method. Determinants are thus only presented for DED calculated by including energy and weight of all foods and solids in beverages.

Higher mean DED estimates were found to be associated with higher intake of energy $(\mathrm{kJ})$ and percentage of total energy (\%TE) from fat, carbohydrates and total sugar $(P<0.001)$ and lower intakes of protein and dietary fibre $(P<0.001)$ (Table 2). Higher consumption or being a consumer of certain foods was found to be both positively and inversely associated with DED. DED estimates were significantly lower in those with higher intakes (g/10 MJ) of vegetables, fruit, potatoes (boiled, baked or mashed), fresh meat, brown bread, fish, vegetable and pulse dishes, eggs, pulses, soup, cooked breakfast cereals and nuts $(P<0 \cdot 001)$. Mean DED estimates were significantly higher in those with higher intakes $(\mathrm{g} / 10 \mathrm{MJ})$ of white bread, chips, ready-to-eat

Table 1. Dietary energy density (DED; kJ/g) estimates for the Irish population, aged 5-90 years, by age and sex $(n 2535)$ (Number of participants and medians; mean values and standard deviations)

\begin{tabular}{|c|c|c|c|c|c|c|c|c|c|c|c|}
\hline \multirow[b]{2}{*}{ Sex } & \multirow[b]{2}{*}{ Age group } & \multirow[b]{2}{*}{$n$} & \multicolumn{3}{|c|}{$\begin{array}{l}\mathrm{DED}(\mathrm{kJ} / \mathrm{g}) \\
\text { total diet }\end{array}$} & \multicolumn{3}{|c|}{$\begin{array}{l}\text { DED }(\mathrm{kJ} / \mathrm{g}) \\
\text { food only }\end{array}$} & \multicolumn{3}{|c|}{$\begin{array}{l}\text { DED }(\mathrm{kJ} / \mathrm{g}) \text { including foods } \\
\text { and solids in beverages }\end{array}$} \\
\hline & & & Mean & Median & SD & Mean & Median & SD & Mean & Median & SD \\
\hline \multirow[t]{16}{*}{ Males } & $5-90$ & 1257 & $3 \cdot 85$ & 3.84 & 1.05 & $7 \cdot 86$ & $7 \cdot 84$ & 1.62 & 8.75 & $8 \cdot 76$ & 1.77 \\
\hline & $5-10$ & 218 & 4.59 & 4.59 & 0.71 & $8 \cdot 31$ & $8 \cdot 17$ & 1.60 & $9 \cdot 33$ & $9 \cdot 18$ & 1.63 \\
\hline & $11-15$ & 212 & 4.53 & 4.52 & 0.84 & 8.44 & $8 \cdot 33$ & 1.51 & $9 \cdot 39$ & $9 \cdot 37$ & 1.59 \\
\hline & $16-20$ & 139 & 4.05 & 3.94 & 0.98 & $8 \cdot 20$ & $8 \cdot 27$ & 1.44 & $9 \cdot 41$ & 9.46 & 1.48 \\
\hline & $21-25$ & 72 & 3.39 & $3 \cdot 22$ & 0.92 & $8 \cdot 33$ & 8.47 & 1.61 & $9 \cdot 52$ & $9 \cdot 79$ & 1.91 \\
\hline & $26-30$ & 86 & $3 \cdot 19$ & 3.01 & 0.98 & $7 \cdot 89$ & 7.94 & 1.41 & 8.83 & 9.03 & 1.58 \\
\hline & $31-35$ & 66 & $3 \cdot 39$ & $3 \cdot 24$ & 1.06 & $7 \cdot 71$ & 7.55 & 1.55 & 8.50 & $8 \cdot 27$ & 1.69 \\
\hline & $36-40$ & 63 & 3.37 & $3 \cdot 19$ & 0.97 & 7.84 & $7 \cdot 78$ & 1.68 & 8.65 & 8.56 & 1.81 \\
\hline & $41-45$ & 66 & 3.53 & $3 \cdot 48$ & 1.04 & $7 \cdot 76$ & $7 \cdot 65$ & 1.33 & 8.48 & $8 \cdot 39$ & 1.47 \\
\hline & $46-50$ & 76 & 3.49 & 3.42 & 0.83 & $7 \cdot 40$ & $7 \cdot 33$ & 1.53 & 8.05 & 7.96 & 1.65 \\
\hline & $51-55$ & 64 & $3 \cdot 26$ & 3.22 & 0.83 & $7 \cdot 29$ & 7.04 & 1.30 & 8.05 & 7.87 & 1.31 \\
\hline & $56-60$ & 49 & 3.42 & 3.35 & 0.84 & 6.97 & 6.96 & $1 \cdot 28$ & $7 \cdot 66$ & $7 \cdot 76$ & 1.26 \\
\hline & $61-65$ & 46 & $3 \cdot 13$ & 2.96 & 0.90 & $6 \cdot 71$ & $6 \cdot 83$ & 1.55 & $7 \cdot 28$ & $7 \cdot 40$ & 1.54 \\
\hline & $66-70$ & 37 & $3 \cdot 66$ & $3 \cdot 65$ & 0.99 & 6.93 & $6 \cdot 91$ & 1.72 & 7.44 & 7.06 & 1.77 \\
\hline & $71-75$ & 31 & $3 \cdot 34$ & 3.33 & 0.87 & 6.55 & $6 \cdot 10$ & $1 \cdot 82$ & $7 \cdot 13$ & 6.96 & 1.85 \\
\hline & $>75$ & 32 & 3.37 & 3.40 & 0.88 & $6 \cdot 52$ & 6.48 & 1.43 & $7 \cdot 01$ & 6.88 & 1.45 \\
\hline$P_{\text {trend }} \dagger$ & & & & $<0.001$ & & & $<0.001$ & & & $<0.001$ & \\
\hline \multirow{16}{*}{ Females } & $5-90$ & 1278 & $3 \cdot 55^{\star}$ & 3.49 & $1 \cdot 11$ & $7 \cdot 31^{*}$ & 7.33 & $1 \cdot 77$ & $8 \cdot 07^{*}$ & 8.17 & 1.93 \\
\hline & $5-10$ & 228 & 4.56 & 4.54 & 0.69 & $8 \cdot 11$ & $8 \cdot 08$ & $1 \cdot 29$ & $9 \cdot 07$ & $9 \cdot 04$ & 1.37 \\
\hline & $11-15$ & 211 & $4 \cdot 31$ & 4.29 & 0.76 & $8 \cdot 30$ & $8 \cdot 20$ & $1 \cdot 38$ & $9 \cdot 20$ & $9 \cdot 08$ & 1.30 \\
\hline & $16-20$ & 115 & 3.73 & $3 \cdot 84$ & 0.96 & $7 \cdot 88$ & $7 \cdot 79$ & 1.64 & $8 \cdot 81$ & 8.83 & 1.73 \\
\hline & $21-25$ & 76 & 3.33 & $3 \cdot 21$ & 1.22 & $7 \cdot 74$ & $7 \cdot 68$ & 1.93 & $8 \cdot 60$ & 8.57 & $2 \cdot 12$ \\
\hline & $26-30$ & 76 & $3 \cdot 13$ & 3.04 & $1 \cdot 10$ & $7 \cdot 36$ & $7 \cdot 36$ & 1.59 & 8.09 & $8 \cdot 25$ & 1.80 \\
\hline & $31-35$ & 67 & $3 \cdot 13$ & $2 \cdot 87$ & 1.04 & 7.44 & 7.40 & $1 \cdot 76$ & $8 \cdot 12$ & $8 \cdot 16$ & 1.83 \\
\hline & $36-40$ & 59 & 2.95 & 3.02 & 0.75 & $7 \cdot 16$ & $7 \cdot 12$ & 1.32 & $7 \cdot 86$ & $7 \cdot 73$ & 1.54 \\
\hline & $41-45$ & 87 & $2 \cdot 87$ & $2 \cdot 80$ & 0.83 & 7.04 & 6.99 & 1.73 & 7.67 & $7 \cdot 74$ & 1.81 \\
\hline & $46-50$ & 86 & 2.95 & $2 \cdot 86$ & 0.89 & 6.55 & $6 \cdot 56$ & 1.65 & $7 \cdot 18$ & $7 \cdot 21$ & 1.75 \\
\hline & $51-55$ & 63 & 2.90 & $2 \cdot 76$ & 0.97 & $6 \cdot 22$ & $5 \cdot 96$ & 1.58 & $6 \cdot 72$ & 6.59 & 1.65 \\
\hline & $56-60$ & 48 & $2 \cdot 93$ & $2 \cdot 82$ & 0.82 & $5 \cdot 80$ & $5 \cdot 84$ & 1.47 & $6 \cdot 24$ & $6 \cdot 19$ & 1.58 \\
\hline & $61-65$ & 52 & $2 \cdot 65$ & $2 \cdot 63$ & 0.73 & $5 \cdot 50$ & $5 \cdot 24$ & 1.42 & 5.93 & $5 \cdot 65$ & 1.47 \\
\hline & $66-70$ & 43 & $2 \cdot 79$ & $2 \cdot 81$ & 0.73 & $5 \cdot 67$ & $5 \cdot 67$ & 1.36 & $6 \cdot 23$ & $6 \cdot 25$ & 1.53 \\
\hline & $71-75$ & 32 & 2.96 & $2 \cdot 73$ & 1.08 & 6.07 & 6.03 & 1.58 & $6 \cdot 50$ & $6 \cdot 43$ & 1.66 \\
\hline & $>75$ & 35 & 2.99 & 2.85 & $0 \cdot 70$ & $5 \cdot 82$ & $5 \cdot 77$ & 1.35 & 6.42 & 6.43 & 1.56 \\
\hline$P_{\text {trend }}$ & & & & $<0.001$ & & & $<0.001$ & & & $<0.001$ & \\
\hline
\end{tabular}

* Mean value was significantly different from that of males as calculated using an independent-samples $t$ test $(P<0.05)$.

$\dagger P$ linear trend across age groups as calculated using two-way ANOVA. 
Table 2. Dietary energy density (DED) estimates by tertile of mean daily energy intake (kJ), macronutrient intakes (percentage of total energy, \%TE), and dietary fibre and food group intakes $(\mathrm{g} / 10 \mathrm{MJ} / \mathrm{d})$ in the Irish population ( $\mathrm{n} 2535)$

(Number of participants, mean values and standard deviations)

\begin{tabular}{|c|c|c|c|c|c|c|c|c|c|c|c|c|c|}
\hline & \multicolumn{9}{|c|}{ DED $(\mathrm{kJ} / \mathrm{g})$ including foods and solids in beverages } & \multirow[b]{2}{*}{$P^{\star}$} & \multirow[b]{2}{*}{$\eta^{2}$} & & \multirow[b]{2}{*}{ Mean intake } \\
\hline & $n$ & Mean & SD & $n$ & Mean & SD & $n$ & Mean & SD & & & & \\
\hline & \multicolumn{3}{|c|}{ Low consumer } & \multicolumn{3}{|c|}{ Medium consumer } & \multicolumn{3}{|c|}{ High consumer } & & & & \\
\hline Energy & 845 & $7.96^{\mathrm{a}}$ & 1.97 & 845 & $8.47^{\mathrm{b}}$ & 1.89 & 845 & $8.87^{\circ}$ & 1.69 & 0.000 & 0.04 & $\uparrow$ & 8094 kJ \\
\hline Protein & 845 & $9.47^{\mathrm{a}}$ & 1.71 & 845 & $8.53^{\mathrm{b}}$ & 1.60 & 845 & $7 \cdot 30^{\mathrm{c}}$ & 1.70 & 0.000 & 0.22 & $\downarrow$ & $15.85 \% \mathrm{TE}$ \\
\hline Fat & 845 & $7.94^{\mathrm{a}}$ & 1.96 & 845 & $8.52^{\mathrm{b}}$ & 1.77 & 845 & $8.83^{\mathrm{c}}$ & 1.83 & 0.000 & 0.04 & $\uparrow$ & $34.67 \% \mathrm{TE}$ \\
\hline Carbohydrates & 845 & $8 \cdot 30^{\mathrm{a}}$ & 1.89 & 845 & $8 \cdot 34^{\mathrm{a}}$ & 1.87 & 845 & $8.65^{\mathrm{b}}$ & 1.89 & 0.000 & 0.01 & $\uparrow$ & $46.06 \% \mathrm{TE}$ \\
\hline Total sugar & 845 & $8.32^{\mathrm{a}}$ & 1.72 & 845 & $8 \cdot 23^{\mathrm{a}}$ & 1.89 & 845 & $8.74^{\mathrm{b}}$ & 2.01 & 0.000 & 0.01 & $\uparrow$ & $19.12 \% \mathrm{TE}$ \\
\hline \multirow[t]{2}{*}{ Dietary fibre } & 845 & $9 \cdot 76^{a}$ & 1.49 & 845 & $8 \cdot 61^{\mathrm{b}}$ & $1 \cdot 41$ & 845 & $6.93^{\mathrm{c}}$ & 1.57 & 0.000 & 0.38 & $\downarrow$ & $21 \cdot 22 \mathrm{~g} / 10 \mathrm{MJ}$ \\
\hline & \multicolumn{3}{|c|}{ Low consumer } & \multicolumn{3}{|c|}{ Medium consumer } & \multicolumn{3}{|c|}{ High consumer } & & & & \\
\hline Vegetables (excluding pulses and composite meals) & 845 & $9.67^{\mathrm{a}}$ & 1.63 & 845 & $8.57^{\mathrm{b}}$ & 1.48 & 845 & $7.05^{\mathrm{c}}$ & 1.56 & 0.000 & 0.32 & $\downarrow$ & $79.3 \mathrm{~g} / 10 \mathrm{MJ}$ \\
\hline Fruit & 845 & $9 \cdot 37^{\mathrm{a}}$ & 1.79 & 845 & $8 \cdot 64^{\mathrm{b}}$ & 1.62 & 845 & $7.29^{c}$ & 1.64 & 0.000 & 0.21 & $\downarrow$ & $109.8 \mathrm{~g} / 10 \mathrm{MJ}$ \\
\hline Potatoes (boiled, baked or mashed) & 845 & $9.08^{\mathrm{a}}$ & 1.98 & 845 & $8.56^{\mathrm{b}}$ & 1.72 & 845 & $7.66^{\mathrm{c}}$ & 1.68 & 0.000 & 0.10 & $\downarrow$ & $88.4 \mathrm{~g} / 10 \mathrm{MJ}$ \\
\hline White bread and rolls & 845 & $7.64^{\mathrm{a}}$ & 1.93 & 845 & $8.74^{\mathrm{b}}$ & 1.85 & 845 & $8.92^{\mathrm{b}}$ & 1.62 & 0.000 & 0.09 & $\uparrow$ & $70.0 \mathrm{~g} / 10 \mathrm{MJ}$ \\
\hline Chipped, fried and roasted potatoes & 845 & $7.72^{\mathrm{a}}$ & 1.96 & 845 & $8.56^{\mathrm{b}}$ & 1.77 & 845 & $9.02^{c}$ & 1.70 & 0.000 & 0.08 & $\uparrow$ & $54.2 \mathrm{~g} / 10 \mathrm{MJ}$ \\
\hline Fresh meat and meat dishes & 845 & $9.04^{\mathrm{a}}$ & $2 \cdot 10$ & 845 & $8.48^{\mathrm{b}}$ & 1.67 & 845 & $7.77^{\mathrm{C}}$ & 1.66 & 0.000 & 0.07 & $\downarrow$ & $133.1 \mathrm{~g} / 10 \mathrm{MJ}$ \\
\hline Ready-to-eat breakfast cereals (including milk) & 845 & $7.89^{\mathrm{a}}$ & 1.99 & 845 & $8.33^{\mathrm{b}}$ & 1.79 & 845 & $9.08^{\mathrm{C}}$ & 1.68 & 0.000 & 0.07 & $\uparrow$ & $224.4 \mathrm{~g} / 10 \mathrm{MJ}$ \\
\hline Processed meat & 845 & $7.96^{\mathrm{a}}$ & 1.99 & 845 & $8.56^{\mathrm{b}}$ & 1.75 & 845 & $8 \cdot 77^{\mathrm{b}}$ & 1.84 & 0.000 & 0.03 & $\uparrow$ & $64.7 \mathrm{~g} / 10 \mathrm{MJ}$ \\
\hline Biscuits (including crackers) & 845 & $8 \cdot 30^{\mathrm{a}}$ & 1.93 & 845 & $8 \cdot 53^{b}$ & 1.85 & 845 & $8.46^{a, b}$ & 1.89 & 0.040 & 0.00 & $\uparrow$ & $16.1 \mathrm{~g} / 10 \mathrm{MJ}$ \\
\hline Butters, spreading fats and oils & 845 & $8.43^{\mathrm{a}}$ & 1.91 & 845 & $8 \cdot 54^{\mathrm{a}, \mathrm{b}}$ & 1.88 & 845 & $8 \cdot 31^{\mathrm{a}, \mathrm{c}}$ & 1.88 & 0.045 & 0.00 & [?] & $15.0 \mathrm{~g} / 10 \mathrm{MJ}$ \\
\hline \multirow[t]{2}{*}{ Sugars, syrups, preserves and sweeteners } & 845 & 8.41 & 1.91 & 845 & 8.54 & 1.82 & 845 & 8.34 & 1.93 & 0.097 & 0.00 & - & $11.2 \mathrm{~g} / 10 \mathrm{MJ}$ \\
\hline & \multicolumn{3}{|c|}{ Non-consumer } & \multicolumn{3}{|c|}{ Low consumer } & \multicolumn{3}{|c|}{ High consumer } & & & & \\
\hline Sugar-sweetened beverages & 979 & $7 \cdot 40^{\mathrm{a}}$ & 1.77 & 777 & $8 \cdot 81^{\mathrm{b}}$ & 1.60 & 779 & $9.34^{\mathrm{C}}$ & 1.68 & 0.000 & 0.20 & $\uparrow$ & $319.4 \mathrm{~g} / 10 \mathrm{MJ}$ \\
\hline Savoury snacks (e.g. crisps and popcorn) & 1162 & $7 \cdot 71^{\mathrm{a}}$ & 1.90 & 686 & $8 \cdot 84^{\mathrm{b}}$ & 1.66 & 687 & $9 \cdot 24^{\mathrm{C}}$ & 1.62 & 0.000 & 0.13 & $\uparrow$ & $11.0 \mathrm{~g} / 10 \mathrm{MJ}$ \\
\hline Brown bread & 995 & $9.07^{\mathrm{a}}$ & 1.76 & 770 & $8.51^{\mathrm{b}}$ & 1.81 & 770 & $7.53^{\mathrm{c}}$ & 1.78 & 0.000 & 0.11 & $\downarrow$ & $46.6 \mathrm{~g} / 10 \mathrm{MJ}$ \\
\hline Fish and fish dishes & 1287 & $8 \cdot 84^{\mathrm{a}}$ & 1.86 & 624 & $8.66^{\mathrm{a}}$ & 1.63 & 624 & $7 \cdot 37^{\mathrm{b}}$ & 1.80 & 0.000 & 0.11 & $\downarrow$ & $41.8 \mathrm{~g} / 10 \mathrm{MJ}$ \\
\hline Chocolate confectionery & 953 & $7 \cdot 77^{\mathrm{a}}$ & 1.90 & 791 & $8 \cdot 50^{\mathrm{b}}$ & 1.73 & 791 & $9 \cdot 16^{\mathrm{C}}$ & 1.76 & 0.000 & 0.09 & $\uparrow$ & $16 \cdot 2 \mathrm{~g} / 10 \mathrm{MJ}$ \\
\hline Non-chocolate confectionery & 1448 & $8.05^{a}$ & 1.92 & 543 & $8.87^{\mathrm{b}}$ & 1.69 & 544 & $9 \cdot 02^{\mathrm{b}}$ & 1.74 & 0.000 & 0.06 & $\uparrow$ & $9.5 \mathrm{~g} / 10 \mathrm{MJ}$ \\
\hline Vegetable and pulse dishes & 1644 & $8.66^{\mathrm{a}}$ & 1.92 & 445 & $8.49^{\mathrm{a}}$ & 1.63 & 446 & $7.54^{\mathrm{b}}$ & 1.78 & 0.000 & 0.05 & $\downarrow$ & $16.7 \mathrm{~g} / 10 \mathrm{MJ}$ \\
\hline Savouries (e.g. pasta dishes and pizzas) & 1116 & $7 \cdot 98^{\mathrm{a}}$ & 2.01 & 709 & $8.76^{\mathrm{b}}$ & 1.79 & 710 & $8.81^{\mathrm{b}}$ & 1.63 & 0.000 & 0.04 & $\uparrow$ & $39.6 \mathrm{~g} / 10 \mathrm{MJ}$ \\
\hline Eggs and egg dishes & 1340 & $8.56^{\mathrm{a}}$ & 1.90 & 597 & $8.77^{\mathrm{a}}$ & 1.79 & 598 & $7 \cdot 80^{\mathrm{b}}$ & 1.82 & 0.000 & 0.04 & $\downarrow$ & $26.4 \mathrm{~g} / 10 \mathrm{MJ}$ \\
\hline Pulses (excluding composite dishes) & 1134 & $8 \cdot 61^{\mathrm{a}}$ & 2.03 & 700 & $8.68^{a}$ & 1.68 & 701 & $7.90^{\mathrm{b}}$ & 1.76 & 0.000 & 0.03 & $\downarrow$ & $19.3 \mathrm{~g} / 10 \mathrm{MJ}$ \\
\hline Rice, pasta and noodles & 1193 & $8.52^{\mathrm{a}}$ & 2.05 & 671 & $8.75^{\mathrm{b}}$ & 1.63 & 671 & $7.95^{\mathrm{c}}$ & 1.75 & 0.000 & 0.03 & 回 & $39.1 \mathrm{~g} / 10 \mathrm{MJ}$ \\
\hline Alcoholic beverages & 1657 & $8.57^{\mathrm{a}}$ & 1.88 & 438 & $7.78^{\mathrm{b}}$ & 1.90 & 440 & $8.54^{\mathrm{a}}$ & 1.79 & 0.000 & 0.02 & 国 & $542.6 \mathrm{~g} / 10 \mathrm{MJ}$ \\
\hline Creams, ice creams and chilled desserts & 1110 & $8.36^{\mathrm{a}}$ & 2.00 & 712 & $8.73^{\mathrm{b}}$ & 1.75 & 713 & $8 \cdot 24^{\mathrm{a}}$ & 1.82 & 0.000 & 0.01 & ] & $28.2 \mathrm{~g} / 10 \mathrm{MJ}$ \\
\hline Yogurts & 1292 & $8.45^{\mathrm{a}}$ & 2.00 & 621 & $8.67^{\mathrm{b}}$ & 1.81 & 622 & $8 \cdot 14^{\mathrm{C}}$ & 1.70 & 0.000 & 0.01 & ] & $42.4 \mathrm{~g} / 10 \mathrm{MJ}$ \\
\hline Cakes, pastries and buns & 1302 & $8.39^{\mathrm{a}}$ & 1.96 & 616 & $8 \cdot 70^{\mathrm{b}}$ & 1.75 & 617 & $8 \cdot 26^{\mathrm{a}}$ & 1.85 & 0.000 & 0.01 & ] & $17.4 \mathrm{~g} / 10 \mathrm{MJ}$ \\
\hline Cheeses & 948 & 8.41 & 2.04 & 793 & 8.52 & 1.76 & 794 & 8.36 & 1.84 & 0.244 & 0.00 & - & $14.2 \mathrm{~g} / 10 \mathrm{MJ}$ \\
\hline \multirow[t]{2}{*}{ Juices and smoothies } & 1247 & 8.34 & 2.02 & 644 & 8.49 & 1.83 & 644 & 8.54 & 1.68 & 0.060 & 0.00 & - & $197.0 \mathrm{~g} / 10 \mathrm{MJ}$ \\
\hline & \multicolumn{3}{|c|}{ Non-consumer } & \multicolumn{3}{|c|}{ Consumer } & & & & & & & \\
\hline Soups & 1760 & 8.66 & 1.89 & 775 & 7.91 & 1.80 & & & & 0.000 & 0.03 & $\downarrow$ & $32.3 \mathrm{~g} / 10 \mathrm{MJ}$ \\
\hline Cooked breakfast cereals (e.g. porridge) & 1953 & 8.54 & 1.90 & 582 & 8.07 & 1.82 & & & & 0.000 & 0.01 & $\downarrow$ & $37.0 \mathrm{~g} / 10 \mathrm{MJ}$ \\
\hline Potato products & 2015 & 8.34 & 1.94 & 520 & 8.80 & 1.64 & & & & 0.000 & 0.01 & $\uparrow$ & $7.0 \mathrm{~g} / 10 \mathrm{MJ}$ \\
\hline Nuts & 2258 & 8.48 & 1.89 & 277 & 8.02 & 1.84 & & & & 0.000 & 0.01 & $\downarrow$ & $1.5 \mathrm{~g} / 10 \mathrm{MJ}$ \\
\hline
\end{tabular}

$\uparrow$ and $\downarrow$, the direction of change in DED as the dietary intakes increase from low to high; 国, a non-directional association of DED with dietary intakes; -, no significant difference in DED with change in dietary intake.

${ }_{a, b, c, c}$ Mean values with unlike superscript letters were significantly different between the consumer groups $(P<0.05)$.

*As calculated using a one-way ANOVA with Tukey's honestly significant difference or Hochberg's GT2 post hoc, or an independent-samples $t$ test. 
Table 3. Dietary energy density (DED) estimates and mean daily energy intake (kJ), macronutrient intakes (percentage of total energy, \%TE), and dietary fibre and food group intakes ( $g / 10 \mathrm{MJ}$ ) in the Irish population by age group split by sex ( $n$ 2535)

(Mean values and standard deviations)

\begin{tabular}{|c|c|c|c|c|c|c|c|c|c|c|c|c|c|c|c|c|}
\hline & \multicolumn{8}{|c|}{ Males } & \multicolumn{8}{|c|}{ Females } \\
\hline & \multicolumn{2}{|c|}{$\begin{array}{l}5-18 \text { years } \\
(n 534)\end{array}$} & \multicolumn{2}{|c|}{$\begin{array}{l}19-50 \text { years } \\
\quad(n 464)\end{array}$} & \multicolumn{2}{|c|}{$\begin{array}{l}51-90 \text { years } \\
\quad(n 259)\end{array}$} & \multirow[b]{2}{*}{$P^{*}$} & & \multicolumn{2}{|c|}{$\begin{array}{l}5-18 \text { years } \\
(n 527)\end{array}$} & \multicolumn{2}{|c|}{$\begin{array}{l}19-50 \text { years } \\
\quad(n 478)\end{array}$} & \multicolumn{2}{|c|}{$\begin{array}{l}51-90 \text { years } \\
\quad(n 273)\end{array}$} & \multirow[b]{2}{*}{$P^{\star}$} & \\
\hline & Mean & SD & Mean & SD & Mean & SD & & & Mean & SD & Mean & SD & Mean & SD & & \\
\hline $\begin{array}{l}\mathrm{DED}(\mathrm{kJ} / \mathrm{g}) \text { including foods and } \\
\text { solids in beverages }\end{array}$ & $9 \cdot 38^{\mathrm{a}}$ & 1.60 & $8 \cdot 71^{\mathrm{b}}$ & $1 \cdot 71$ & $7 \cdot 51^{\mathrm{C}}$ & 1.53 & 0.000 & $\downarrow$ & $9.07^{a}$ & 1.40 & $7.95^{\mathrm{b}}$ & 1.89 & $6 \cdot 34^{\mathrm{C}}$ & 1.58 & 0.000 & $\downarrow$ \\
\hline Energy $(\mathrm{kJ})$ & $8386^{\mathrm{a}}$ & 2303 & $10266^{\mathrm{b}}$ & 2777 & $8911^{\mathrm{c}}$ & 2563 & 0.000 & 回 & $6864^{\mathrm{a}}$ & 1586 & $7302^{\mathrm{b}}$ & 2094 & $6819^{\mathrm{a}}$ & 1701 & 0.000 & ] \\
\hline Protein (\%TE) & $14 \cdot 3^{\mathrm{a}}$ & 2.5 & $17 \cdot 1^{\mathrm{b}}$ & 4.0 & $17.4^{\mathrm{b}}$ & 3.6 & 0.000 & $\uparrow$ & $13 \cdot 9^{\mathrm{a}}$ & $2 \cdot 3$ & $16 \cdot 4^{\mathrm{b}}$ & 3.7 & $17 \cdot 9^{\mathrm{c}}$ & 3.4 & 0.000 & $\uparrow$ \\
\hline Fat (\%TE) & $34 \cdot 3$ & 4.9 & $34 \cdot 2$ & $6 \cdot 1$ & 34.7 & $7 \cdot 1$ & 0.618 & - & $35 \cdot 0$ & 4.4 & $35 \cdot 0$ & 6.0 & 34.8 & $6 \cdot 1$ & 0.858 & - \\
\hline Carbohydrates (\%TE) & $50 \cdot 6^{\mathrm{a}}$ & 5.7 & $41 \cdot 6^{\mathrm{b}}$ & $7 \cdot 3$ & $42 \cdot 2^{\mathrm{b}}$ & 7.2 & 0.000 & $\downarrow$ & $50 \cdot 5^{\mathrm{a}}$ & 4.8 & $43 \cdot 6^{\mathrm{b}}$ & 6.4 & $44.4^{\mathrm{b}}$ & $6 \cdot 3$ & 0.000 & $\downarrow$ \\
\hline Total sugar (\%TE) & $22 \cdot 3^{\mathrm{a}}$ & 5.7 & $15 \cdot 6^{\mathrm{b}}$ & $5 \cdot 6$ & $16 \cdot 2^{\mathrm{b}}$ & 6.3 & 0.000 & $\downarrow$ & $22 \cdot 3^{\mathrm{a}}$ & $5 \cdot 3$ & $17 \cdot 3^{\mathrm{b}}$ & 5.5 & $18.7^{\mathrm{c}}$ & $5 \cdot 6$ & 0.000 & $\uparrow$ \\
\hline Dietary fibre $(\mathrm{g} / 10 \mathrm{MJ})$ & $18 \cdot 2^{\mathrm{a}}$ & 5.0 & $20.9^{\mathrm{b}}$ & 7.4 & $23 \cdot 2^{\mathrm{c}}$ & 7.4 & 0.000 & $\uparrow$ & $18 \cdot 2^{\mathrm{a}}$ & 4.6 & $23 \cdot 3^{\mathrm{b}}$ & 7.5 & $28 \cdot 1^{\mathrm{c}}$ & 8.6 & 0.000 & $\uparrow$ \\
\hline $\begin{array}{l}\text { Vegetables (excluding pulses and composite } \\
\text { meals) (g/10 MJ) }\end{array}$ & $41 \cdot 1^{\mathrm{a}}$ & 42.7 & $69.5^{\mathrm{b}}$ & 64.8 & $104 \cdot 7^{c}$ & 86.8 & 0.000 & $\uparrow$ & $46 \cdot 8^{\mathrm{a}}$ & 49.8 & $113 \cdot 5^{\mathrm{b}}$ & $101 \cdot 1$ & $149 \cdot 9^{c}$ & 109.8 & 0.000 & $\uparrow$ \\
\hline Fruit $(\mathrm{g} / 10 \mathrm{MJ})$ & $89.9^{\mathrm{a}}$ & $136 \cdot 7$ & $83.4^{\mathrm{a}}$ & $95 \cdot 8$ & $114 \cdot 7^{\mathrm{b}}$ & 128.9 & 0.000 & $\uparrow$ & $103 \cdot 0^{\mathrm{a}}$ & 103.5 & $117 \cdot 2^{\mathrm{a}}$ & $135 \cdot 3$ & $189 \cdot 3^{\mathrm{b}}$ & $171 \cdot 3$ & 0.000 & $\uparrow$ \\
\hline Fish and fish dishes (g/10 MJ) & $12 \cdot 9^{\mathrm{a}}$ & $22 \cdot 2$ & $41 \cdot 2^{\mathrm{b}}$ & 69.4 & $88 \cdot 6^{\mathrm{c}}$ & $109 \cdot 7$ & 0.000 & $\uparrow$ & $13.5^{\mathrm{a}}$ & 24.9 & $58 \cdot 6^{\mathrm{b}}$ & 89.4 & $80 \cdot 2^{\mathrm{c}}$ & 98.6 & 0.000 & $\uparrow$ \\
\hline Potatoes (boiled, baked or mashed) ( $\mathrm{g} / 10 \mathrm{MJ})$ & $80 \cdot 8^{\mathrm{a}}$ & 72.0 & $80.5^{\mathrm{a}}$ & 83.0 & $134 \cdot 4^{\mathrm{b}}$ & 106.9 & 0.000 & $\uparrow$ & $78 \cdot 1^{\mathrm{a}}$ & $70 \cdot 8$ & $73 \cdot 7^{\mathrm{a}}$ & 79.9 & $118.4^{\mathrm{b}}$ & $90 \cdot 6$ & 0.000 & $\uparrow$ \\
\hline Meat and meat dishes (g/10 MJ) & $112 \cdot 0^{\mathrm{a}}$ & $71 \cdot 7$ & $157 \cdot 3^{\mathrm{b}}$ & $110 \cdot 3$ & $144 \cdot 0^{\mathrm{b}}$ & $95 \cdot 1$ & 0.000 & $\uparrow$ & $110 \cdot 8^{\mathrm{a}}$ & 71.5 & $136 \cdot 8^{\mathrm{b}}$ & $101 \cdot 1$ & $159 \cdot 5^{\mathrm{c}}$ & $107 \cdot 9$ & 0.000 & $\uparrow$ \\
\hline Brown bread (g/10 MJ) & $19 \cdot 5^{\mathrm{a}}$ & 38.6 & $55 \cdot 3^{\mathrm{b}}$ & $58 \cdot 8$ & $79 \cdot 5^{\mathrm{c}}$ & 80.0 & 0.000 & $\uparrow$ & $19 \cdot 9^{\mathrm{a}}$ & 32.9 & $60 \cdot 3^{\mathrm{b}}$ & 61.5 & $81 \cdot 0^{\mathrm{c}}$ & 66.3 & 0.000 & $\uparrow$ \\
\hline Sugar-sweetened beverages (g/10 MJ) & $280.5^{\mathrm{a}}$ & $226 \cdot 7$ & $167 \cdot 8^{\mathrm{b}}$ & 284.2 & $58 \cdot 2^{\mathrm{c}}$ & $174 \cdot 0$ & 0.000 & $\downarrow$ & $271 \cdot 2^{\mathrm{a}}$ & 253.0 & $188 \cdot 6^{\mathrm{b}}$ & $364 \cdot 2$ & $77 \cdot 7^{\mathrm{c}}$ & 287.6 & 0.000 & $\downarrow$ \\
\hline Chocolate confectionery (g/10 MJ) & $22 \cdot 8^{\mathrm{a}}$ & 22.5 & $11 \cdot 1^{\mathrm{b}}$ & 16.5 & $5 \cdot 1^{\mathrm{c}}$ & 10.5 & 0.000 & $\downarrow$ & $25 \cdot 4^{\mathrm{a}}$ & $23 \cdot 1$ & $14 \cdot 6^{\mathrm{b}}$ & 19.9 & $7 \cdot 8^{\mathrm{c}}$ & $15 \cdot 9$ & 0.000 & $\downarrow$ \\
\hline $\begin{array}{l}\text { Ready-to-eat breakfast cereals (including milk) } \\
(\mathrm{g} / 10 \mathrm{MJ})\end{array}$ & $415 \cdot 3^{a}$ & $252 \cdot 3$ & $127 \cdot 2^{b}$ & 130.1 & $84.0^{\circ}$ & $100 \cdot 2$ & 0.000 & $\downarrow$ & $352.4^{a}$ & 250.5 & $108 \cdot 2^{b}$ & $127 \cdot 7$ & $105 \cdot 3^{\mathrm{b}}$ & 124.9 & 0.000 & $\downarrow$ \\
\hline $\begin{array}{l}\text { Savoury snacks (e.g. crisps and popcorn) } \\
(\mathrm{g} / 10 \mathrm{MJ})\end{array}$ & $14 \cdot 2^{\mathrm{a}}$ & $15 \cdot 1$ & $8 \cdot 1^{\mathrm{b}}$ & $13 \cdot 6$ & $1.8^{\mathrm{C}}$ & $8 \cdot 2$ & 0.000 & $\downarrow$ & $20 \cdot 1^{a}$ & $18 \cdot 1$ & $10 \cdot 4^{\mathrm{b}}$ & $15 \cdot 3$ & $2 \cdot 1^{\mathrm{C}}$ & $6 \cdot 1$ & 0.000 & $\downarrow$ \\
\hline Chipped, fried and roasted potatoes ( $\mathrm{g} / 10 \mathrm{MJ}$ ) & $65 \cdot 2^{\mathrm{a}}$ & 53.6 & $52 \cdot 2^{\mathrm{b}}$ & $54 \cdot 0$ & $37.5^{\mathrm{c}}$ & $47 \cdot 9$ & 0.000 & $\downarrow$ & $67.7^{\mathrm{a}}$ & 56.5 & $48.9^{\mathrm{b}}$ & $56 \cdot 2$ & $35.4^{\mathrm{c}}$ & 46.9 & 0.000 & $\downarrow$ \\
\hline White bread ( $g / 10 \mathrm{MJ})$ & $87.6^{\mathrm{a}}$ & 50.6 & $62 \cdot 6^{\mathrm{b}}$ & 56.9 & $69 \cdot 0^{\mathrm{b}}$ & $72 \cdot 4$ & 0.000 & $\downarrow$ & $80 \cdot 8^{\mathrm{a}}$ & $47 \cdot 6$ & $57.9^{\mathrm{b}}$ & 56.9 & $49 \cdot 1^{\mathrm{b}}$ & 62.6 & 0.000 & $\downarrow$ \\
\hline
\end{tabular}

$\uparrow$ and $\downarrow$, the direction of change in DED as the nutrient or food group intakes increase from low to high; 互, a non-directional association of DED with nutrient or food group intakes; -, no significant difference in DED with change in dietary intake.

*As calculated using unlike superscript letters were significantly between the age groups within a sex $(P<0.05)$.

calculated using a one-way ANOVA. 
breakfast cereals (RTEBC), processed meat, sugar-sweetened beverages (SSB), savoury snacks, chocolate and nonchocolate confectionery, savouries, alcohol and potato products $(P<0 \cdot 001)$. Variation in the intakes of vegetables, fruit and SSB $(\mathrm{g} / 10 \mathrm{MJ})$ was found to be associated with the largest differences in mean DED estimates between consumer groups $\left(\eta^{2}\right.$ : vegetables $0 \cdot 32$, fruit $0 \cdot 21$ and SSB $\left.0 \cdot 20\right)$. Differences in the intakes $(\mathrm{g} / 10 \mathrm{MJ})$ of potatoes, fresh meat, brown bread, bananas, fish, white bread, chips, RTEBC, and chocolate and non-chocolate confectionery also contributed to the differences in DED estimates, but to a lesser extent $\left(\eta^{2}: 0 \cdot 06-0 \cdot 13\right)$.

To explain the lower DED estimates obtained for females and the inverse association of DED with age, energy-adjusted intake patterns of macronutrients and dietary fibre and of the food groups that contributed to the largest variation in mean DED estimates were examined across age groups, split by sex (Table 3), and between sexes, adjusted for age (Table 4). Higher intakes of protein, total sugar (\%TE) and dietary fibre $(\mathrm{g} / 10 \mathrm{MJ})$ and lower intakes of carbohydrates (\%TE) were found to be associated with older age groups $(P<0.001)$. The intakes $(\mathrm{g} / 10 \mathrm{MJ})$ of vegetables, fruit, fish, potatoes, fresh meat and brown bread were significantly higher $(P<0.001)$ in older age groups in males and females, while the intakes $(\mathrm{g} / 10 \mathrm{MJ})$ of SSB, chocolate and non-chocolate confectionery, RTEBC, savoury snacks, chips and white bread were significantly lower $(P<0 \cdot 001)$. The intakes of fat $(P=0.007)$, carbohydrates, total sugar (\%TE) and dietary fibre $(\mathrm{g} / 10 \mathrm{MJ})(P<0.001)$ were higher in females than in males, while those of energy $(\mathrm{kJ})(P<0.001)$ and protein (\%TE) $(P=0.003)$ were lower. The intakes of vegetables, fruit, chocolate confectionery and savoury snacks were significantly higher in females than in males $(P<0.001)$ and those of potatoes $(P=0.020)$, RTEBC $(P=0.001)$ and white bread $(P<0.001)$ were significantly higher in males than in females.

The DED (calculated including energy and weight of all foods and solids in beverages) estimates of the NSIFCS participants were also inversely associated with age group $(P<0.001)$ and females had lower DED estimates than males $(P<0.001)$. Mean DED was $8.50($ SD 1.55$) \mathrm{kJ} / \mathrm{g}$ for males and $7 \cdot 10$ (SD 1.59$) \mathrm{kJ} / \mathrm{g}$ for females. Comparison of DED estimates of the sample from the NSIFCS and the comparable subsample from the NANS, split by age and sex, is shown in Fig. 1. The mean DED estimate obtained for women, adjusted for age, in the NSIFCS did not differ significantly from that obtained for the subsample in the NANS $(P=0 \cdot 427)$. However, DED estimates obtained for men in the NSIFCS were significantly lower than those obtained for men in the NANS $(P<0 \cdot 001)$ over the 10 years, differences were apparent in only two age groups: $41-45(P=0 \cdot 015)$ and $56-60(P=0 \cdot 019)$ years.

\section{Discussion}

In the nationally representative free-living Irish population aged 5-90 years included in the present study, DED estimates were higher for males than for females and were inversely associated with age group. Little difference was observed in DED estimates calculated for adults between the two surveys carried out 10 years apart. The intakes of fruit, vegetables and SSB were the major dietary determinants of DED in this population.

Many methods have been proposed for the calculation of DED and there is little consensus as to which is the most appropriate method for free-living populations ${ }^{(35-37)}$. Part of the difficulty lies in the high dependency of DED on the amount of water in the diet. The treatment of beverages is

Table 4. Dietary energy density (DED) estimates and mean daily energy intake (kJ), macronutrient intake (percentage of total energy, \%TE), and dietary fibre and food group intakes (g/10 MJ) in the Irish population by sex adjusted for age (n 2535)

(Mean values with their standard errors)

\begin{tabular}{|c|c|c|c|c|c|}
\hline & \multicolumn{2}{|c|}{ Males } & \multicolumn{2}{|c|}{ Females } & \multirow[b]{2}{*}{$P^{*}$} \\
\hline & Mean & SE & Mean & SE & \\
\hline DED $(\mathrm{kJ} / \mathrm{g})$ including foods and solids in beverages & 8.73 & 0.05 & 8.09 & 0.05 & 0.000 \\
\hline Energy $(\mathrm{kJ})$ & 9190 & 64 & 7016 & 64 & 0.000 \\
\hline Protein (\%TE) & $16 \cdot 0$ & $0 \cdot 1$ & $15 \cdot 7$ & $0 \cdot 1$ & 0.003 \\
\hline Fat $(\%$ TE) & $34 \cdot 4$ & $0 \cdot 2$ & $35 \cdot 0$ & $0 \cdot 2$ & 0.007 \\
\hline Carbohydrates (\%TE) & $45 \cdot 5$ & $0 \cdot 2$ & $46 \cdot 6$ & $0 \cdot 2$ & 0.000 \\
\hline Total sugar (\%TE) & $18 \cdot 5$ & $0 \cdot 2$ & $19 \cdot 7$ & $0 \cdot 2$ & 0.000 \\
\hline Dietary fibre $(\mathrm{g} / 10 \mathrm{MJ})$ & $20 \cdot 3$ & $0 \cdot 2$ & $22 \cdot 1$ & $0 \cdot 2$ & 0.000 \\
\hline Vegetables (excluding pulses and composite meals) (g/10 MJ) & $65 \cdot 4$ & $2 \cdot 1$ & $93 \cdot 0$ & $2 \cdot 1$ & 0.000 \\
\hline Fruit $(g / 10 \mathrm{MJ})$ & $93 \cdot 0$ & $3 \cdot 6$ & $126 \cdot 3$ & $3 \cdot 6$ & 0.000 \\
\hline Fish and fish dishes $(\mathrm{g} / 10 \mathrm{MJ})$ & $39 \cdot 6$ & $2 \cdot 0$ & $44 \cdot 0$ & $2 \cdot 0$ & 0.106 \\
\hline Potatoes (boiled, baked or mashed) (g/10 MJ) & $92 \cdot 2$ & $2 \cdot 3$ & $84 \cdot 7$ & $2 \cdot 3$ & 0.020 \\
\hline Meat and meat dishes (g/10 MJ) & $135 \cdot 7$ & $2 \cdot 6$ & $130 \cdot 5$ & $2 \cdot 6$ & 0.159 \\
\hline Brown bread (g/10 MJ) & $45 \cdot 6$ & 1.5 & 47.5 & 1.5 & 0.376 \\
\hline Sugar-sweetened beverages (g/10 MJ) & $191 \cdot 1$ & $7 \cdot 7$ & $200 \cdot 9$ & $7 \cdot 6$ & 0.363 \\
\hline Chocolate confectionery $(\mathrm{g} / 10 \mathrm{MJ})$ & $14 \cdot 7$ & 0.6 & $17 \cdot 7$ & 0.5 & 0.000 \\
\hline Ready-to-eat breakfast cereals (including milk) (g/10 MJ) & $238 \cdot 1$ & $5 \cdot 7$ & $210 \cdot 8$ & $5 \cdot 6$ & 0.001 \\
\hline Savoury snacks (e.g. crisps and popcorn) (g/10 MJ) & $9 \cdot 3$ & 0.4 & $12 \cdot 8$ & 0.4 & 0.000 \\
\hline Chipped, fried and roasted potatoes (g/10 MJ) & $54 \cdot 4$ & 1.5 & $54 \cdot 0$ & 1.5 & 0.841 \\
\hline White bread (g/10 MJ) & $74 \cdot 3$ & 1.6 & $65 \cdot 7$ & $1 \cdot 6$ & 0.000 \\
\hline
\end{tabular}

*As calculated using a one-way ANCOVA. 


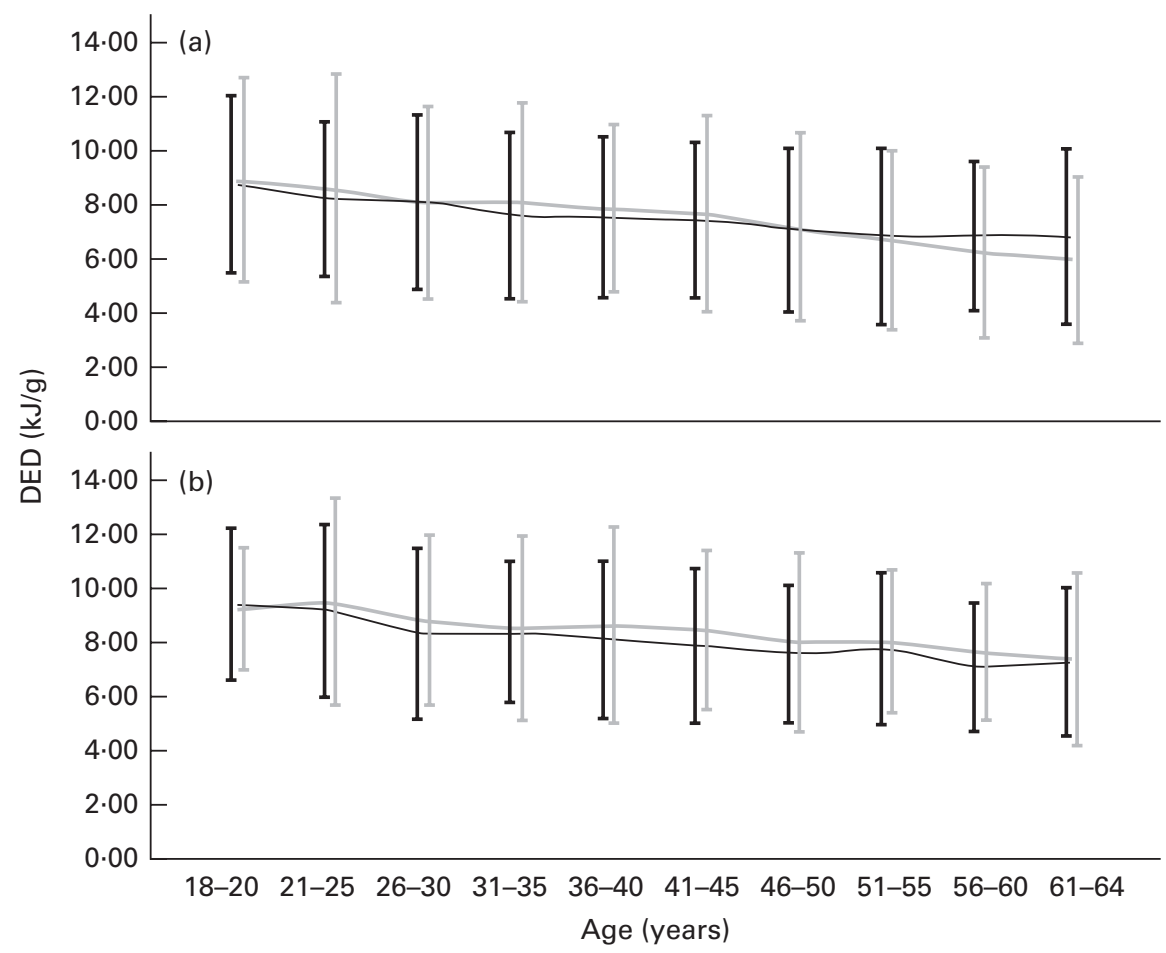

Fig. 1. Comparison of dietary energy density (DED) estimates $(\mathrm{kJ} / \mathrm{g})$ calculated including solids in beverages for the lrish adult population aged $18-64$ years, by age and sex ((a) females and (b) males) in the North/South Ireland Food Consumption Survey (-) and the National Adult Nutrition Survey (-). Error bars: \pm 2 sD.

therefore important, as the inclusion of beverages has a lowering effect on the resulting DED estimates ${ }^{(37)}$. Variation in the associations of dietary and anthropometric factors with DED, depending on the method of computation, has been acknowledged ${ }^{(9,14,35)}$. Johnson et al. ${ }^{(38)}$ proposed calculating DED by excluding drinks and including energy contributed by drinks as a covariate in analyses as the most reliable method for testing the relationship between DED and weight gain in free-living humans. The energy derived from beverages may be an important factor in the causation of obesity, in particular, from SSB, which have been reported to be independently associated with obesity ${ }^{(39)}$. In the present study, nationally representative estimates of DED calculated using three different calculation methods were evaluated. Although the DED estimates differed, the determinants and age-sex trends did not. As such, the primary analysis was focused on DED estimates calculated using a novel method: including energy and weight of all foods and solids in beverages. This approach aims to remove the bias that including beverages can introduce in DED estimates while taking into account the contribution of beverages to EI and as such has potential as a standardised method for further publications.

There was little difference in mean DED estimates on including and excluding UR. Trends in DED estimates across sexes and age groups were constant irrespective of accuracy in reporting energy. Other studies have also reported energy under-reporting as having no substantial effects on DED estimates $^{(10,16)}$. Thus, the trends in DED estimates with population demographics and associations of dietary intake with DED were investigated by including all participants.

It is difficult to compare these estimates with published estimates from other populations as the methods used for calculating DED vary considerably, as discussed above. This further highlights the need for a standardised method. The DED estimates calculated for the Irish population using the food only method were found to be remarkably similar to those calculated for a sample from Northern Ireland ${ }^{(40)}$, higher than those for a sample from Spain ${ }^{(10)}$ and lower than those for a sample from the USA ${ }^{(37)}$. Some consistent trends were observed across the data; females have been reported to have lower DED estimates in child and adult groups in a number of studies ${ }^{(7,10,14,16,37)}$; similarly, Irish DED estimates were lower for females than for males. In keeping with the Irish data, a decrease in DED with age has been observed; Mendoza et al. ${ }^{(7)}$ reported a peak in DED at the age of 7-8 years and a decline thereafter in US children, while Kant \& Graubard ${ }^{(14)}$ reported a lower mean DED in US adults aged $>50$ years than in those aged $\leq 50$ years, and Ledikwe et al $^{(37)}$ found that those aged $\geq 70$ years had significantly lower DED estimates than other age groups.

DED is primarily determined by the water and fat contents of the diet ${ }^{(41)}$. Studies have shown a positive association of EI with $\operatorname{DED}^{(2)}$ and associations of lower DED with higher fat, added sugar and dietary fibre intakes and lower protein and carbohydrate intakes ${ }^{(14,42)}$. Fruit and vegetables are the food groups most commonly associated with lower DED in the literature ${ }^{(8,14,16)}$. In the present study, higher intakes of energy (kJ), fat (\%TE), carbohydrates (\%TE) and total sugar (\%TE) were found to be associated with higher DED estimates, while higher intakes of protein (\%TE) and dietary fibre $(\mathrm{g} / 10 \mathrm{MJ})$ were found to be associated with lower DED estimates. Variation in the intakes of fruit, vegetables and SSB had the greatest effect on mean DED estimates, followed 
by that in the intakes of potatoes, fresh meat, brown bread, white bread, chips, RTEBC, and chocolate and non-chocolate confectionery. Higher intakes of fruit, vegetables, potatoes, fresh meat and brown bread were associated with lower DED estimates, while higher intakes of SSB, white bread, chips, RTEBC, and chocolate and non-chocolate confectionery were associated with higher DED estimates.

In an attempt to explain the lower DED estimates obtained for females and the inverse association of DED estimates with age group, energy-adjusted intake patterns of nutrients and the food groups that explained large amounts of variance in DED estimates in the population as a whole were examined across sexes and age groups. As DED decreased across age groups, intakes of all dietary components examined that were associated with higher DED estimates in the total population decreased and those associated with lower DED estimates increased. In particular, the intakes of fruit and vegetables increased and those of RTEBC decreased considerably across age groups, with the intakes of vegetables in 5- to 18-year-olds more than doubling when compared with those in the 51-90 years age group and those of RTEBC decreasing 3-fold. The disparity in DED estimates between sexes is less clearly explained by differences in consumption patterns. Females consumed significantly more fat, carbohydrates, total sugar, dietary fibre, fruit, vegetables, chocolate confectionery and savoury snacks and less energy, protein, potatoes and RTEBC when compared with males. Of particular interest is that females consumed 1.5 times more fruit and vegetables combined when compared with males.

The downward trend in DED estimates with age group should not be interpreted as longitudinal variations in DED, as the present analysis is based on cross-sectional data and generational effects are possible. However, to examine the potential generational effect, estimates calculated for adults from the NSIFCS were compared with those calculated for a subsample, comparable for age and sex, of adults from the NANS. Little difference was observed in Irish DED estimates over time, adding credibility to the presence of an inverse association between DED and age group.

In conclusion, differences in food choices, in particular, fruit, vegetables and RTEBC, explain the variation in DED estimates by age group and sex. The intakes of fruit, vegetables and SSB appear to have the greatest influence on DED in the Irish population and are potential targets for public health interventions. DED estimates calculated for Irish adults have not changed appreciably in the last 10 years. The DED estimates calculated in the present study are the first nationally representative estimates for an Irish population and are reported by sex and age group to serve as a baseline for future studies and public health interventions.

\section{Supplementary material}

To view supplementary material for this article, please visit http://dx.doi.org/10.1017/S0007114514003420

\section{Acknowledgements}

The authors thank the participants and their families for taking part in the surveys.

The present study was supported by the Irish Government under the National Development Plan (2000-6) and the Department of Agriculture, Fisheries \& Food under the Food for Health Research Initiative (2007-12)

The authors' contributions are as follows: L. O'C carried out the analysis, drafted the manuscript and is responsible for the integrity of the data; J. W. is the co-ordinator of the national nutrition surveys and contributed to the manuscript content; A. F. is a principal investigator of the national nutrition surveys and provided critical input for the analysis. All authors critically reviewed the manuscript and approved the final version submitted for publication.

None of the authors has any conflicts of interest to declare.

\section{References}

1. Yao M \& Roberts SB (2001) Dietary energy density and weight regulation. Nutr Rev 59, 247-258.

2. Rolls BJ (2009) The relationship between dietary energy density and energy intake. Physiol Behav 97, 609-615.

3. Ledikwe JH, Rolls BJ, Smiciklas-Wright H, et al. (2007) Reductions in dietary energy density are associated with weight loss in overweight and obese participants in the PREMIER trial. Am J Clin Nutr 85, 1212-1221.

4. Vernarelli JA, Mitchell DC, Hartman TJ, et al. (2011) Dietary energy density is associated with body weight status and vegetable intake in U.S. children. J Nutr 141, 2204-2210.

5. Howarth NC, Murphy SP, Wilkens LR, et al. (2006) Dietary energy density is associated with overweight status among 5 ethnic groups in the multiethnic cohort study. J Nutr 136, 2243-2248.

6. Murakami K, Sasaki S, Takahashi Y, et al. (2007) Dietary energy density is associated with body mass index and waist circumference, but not with other metabolic risk factors, in free-living young Japanese women. Nutrition 23, 798-806.

7. Mendoza JA, Drewnowski A \& Christakis DA (2007) Dietary energy density is associated with obesity and the metabolic syndrome in US adults. Diabetes Care 30, 974-979.

8. Ledikwe JH, Blanck HM, Khan LK, et al. (2006) Low-energydensity diets are associated with high diet quality in adults in the United States. J Am Diet Assoc 106, 1172-1180.

9. O'Connor L, Walton J \& Flynn A (2013) Dietary energy density and its association with the nutritional quality of the diet of children and teenagers. J Nutr Sci $\mathbf{2}$, e10.

10. Schroder H, Covas M, Elosua R, et al. (2008) Diet quality and lifestyle associated with free selected low-energy density diets in a representative Spanish population. Eur J Clin Nutr 62, 1194-1200.

11. Schroder H, Vila J, Marrugat J, et al. (2008) Low energy density diets are associated with favorable nutrient intake profile and adequacy in free-living elderly men and women. J Nutr 138, 1476-1481.

12. Cuco G, Arija V, Marti-Henneberg C, et al. (2001) Food and nutritional profile of high energy density consumers in an adult Mediterranean population. Eur J Clin Nutr 55, 192-199.

13. Crowe TC, Fontaine HL, Gibbons CJ, et al. (2004) Energy density of foods and beverages in the Australian food 
supply: influence of macronutrients and comparison to dietary intake. Eur J Clin Nutr 58, 1485-1491.

14. Kant AK \& Graubard BI (2005) Energy density of diets reported by American adults: association with food group intake, nutrient intake, and body weight. Int J Obes (Lond) 29, 950-956.

15. Rolls BJ \& Bell EA (1999) Intake of fat and carbohydrate: role of energy density. Eur J Clin Nutr 53, Suppl. 1, S166-S173.

16. Patterson E, Warnberg J, Poortvliet E, et al. (2010) Dietary energy density as a marker of dietary quality in Swedish children and adolescents: the European Youth Heart Study. Eur J Clin Nutr 64, 356-363.

17. Food Standards Agency (2002) McCance and Widdowson's The Composition of Foods, 6th ed. Cambridge, UK: Royal Society of Chemistry.

18. Holland B, Welch AA, Unwin ID, et al. (1995) McCance $\mathcal{E}$ Widdowson's The Composition of Foods, 5th ed. London: HMSO. (Royal Society of Chemistry and Ministry of Agriculture, Fisheries and Food).

19. Holland B, Brown J \& Buss DH (1993) Fish and Fish Products. Third Supplement to McCance and Widdowson's The Composition of Foods, 5th ed. London: HMSO.

20. Holland B, Unwin ID \& Buss DH (1992) Fruit and Nuts. First Supplement to McCance and Widdowson's The Composition of Foods, 5th ed. London: HMSO.

21. Holland B, Unwin ID \& Buss DH (1991) Vegetables, Herbs and Spices. Fifth Supplement to McCance and Widdowson's The Composition of Foods, 5th ed. London: HMSO.

22. Holland B, Unwin ID \& Buss DH (1989) Milk Products and Eggs. Fourth Supplement to McCance and Widdowson's The Composition of Foods, 4th ed. London: HMSO.

23. Holland B, Unwin ID \& Buss DH (1988) Cereal and Cereal Products. Third Supplement to McCance and Widdowson's The Composition of Foods, 4th ed. London: HMSO.

24. Holland B, Welch AA \& Buss DH (1992) Vegetable Dishes. Second Supplement to McCance and Widdowson's The Composition of Foods, 5th ed. London: HMSO.

25. Chan DS, Lau R, Aune D, et al. (2011) Red and processed meat and colorectal cancer incidence: meta-analysis of prospective studies. PLoS One 6, e20456.

26. Chan W, Brown J \& Buss DH (1994) Miscellaneous Foods. Fourth Supplement to McCance and Widdowson's The Composition of Foods, 5th ed. London: HMSO.

27. Chan W, Brown J, Church SM, et al. (1996) Meat Products and Dishes. Sixth Supplement to McCance and Widdowson's The Composition of Foods, 5th ed. London: HMSO.

28. Chan W, Brown J, Lee S, et al. (1996) Meat, Poultry and Game. Fifth Supplement to McCance and Widdowson's The Composition of Foods, 5th ed. London: HMSO.
29. Black LJ, Ireland J, Moller A, et al. (2011) Development of an on-line Irish food composition database for nutrients. J Food Comp Anal 24, 1017-1023.

30. Schofield WN (1985) Predicting basal metabolic rate, new standards and review of previous work. Hum Nutr Clin Nutr 39, Suppl. 1, 5-41.

31. Torun B, Davies PS, Livingstone MB, et al. (1996) Energy requirements and dietary energy recommendations for children and adolescents 1 to 18 years old. Eur J Clin Nutr 50, Suppl. 1, S37-S80 (discussion S80-S81).

32. Goldberg GR, Black AE, Jebb SA, et al. (1991) Criticalevaluation of energy-intake data using fundamental principles of energy physiology. 1. Derivation of cutoff limits to identify under-recording. Eur J Clin Nutr 45, 569-581.

33. Cohen JW (1998) The analysis of variance. In Statistical Power Analysis for the Behavioural Sciences, chapter 8, 2nd ed. Hillside, NJ: Lawrence Erlbaum Associates.

34. Harrington KE, Robson PJ, Kiely M, et al. (2001) The North/ South Ireland Food Consumption Survey: survey design and methodology. Public Health Nutr 4, 1037-1042.

35. Cox DN \& Mela DJ (2000) Determination of energy density of freely selected diets: methodological issues and implications. Int J Obes Relat Metab Disord 24, 49-54.

36. Vernarelli JA, Mitchell DC, Rolls BJ, et al. (2013) Methods for calculating dietary energy density in a nationally representative sample. Procedia Food Sci 2, 68-74.

37. Ledikwe JH, Blanck HM, Khan LK, et al. (2005) Dietary energy density determined by eight calculation methods in a nationally representative United States population. $J$ Nutr 135, 273-278.

38. Johnson L, Wilks DC, Lindroos AK, et al. (2009) Reflections from a systematic review of dietary energy density and weight gain: is the inclusion of drinks valid? Obes Rev 10, 681-692.

39. Malik VS, Pan A, Willett WC, et al. (2013) Sugar-sweetened beverages and weight gain in children and adults: a systematic review and meta-analysis. Am J Clin Nutr 98 , 1084-1102.

40. McCaffrey TA, Rennie KL, Kerr MA, et al. (2008) Energy density of the diet and change in body fatness from childhood to adolescence; is there a relation? Am J Clin Nutr 87, 1230-1237.

41. Stubbs J, Ferres S \& Horgan G (2000) Energy density of foods: effects on energy intake. Crit Rev Food Sci Nutr 40, $481-515$.

42. Ledikwe JH, Blanck HM, Kettel Khan L, et al. (2006) Dietary energy density is associated with energy intake and weight status in US adults. Am J Clin Nutr 83, $1362-1368$. 